\title{
The $\boldsymbol{C}_{2 v}$ shape of enolic acetylacetone
}

Walther Caminati and Jens-Uwe Grabow.

\author{
Dipartimento di Chimica "G. Ciamician" dell'Università, Via Selmi 2, I-40126 Bologna, Italy; \\ Institut für Physikalische Chemie und Elektrochemie, Lehrgebiet A, Callinstrasse 3-3a, D-30167 Hannover, Germany \\ Supplementary Material: Tables (2) of frequencies and figures (2) of conformers.
}

Tables:

Table S1: Measured Frequencies $v$ and deviations $\Delta v$ (observed-calculated) of $\mathrm{A}_{1}$ state rotational transitions $J^{\prime}\left(K_{-1}{ }^{\prime}, K_{+1}{ }^{\prime}\right) \leftarrow J J^{\prime}\left(K_{-1}{ }^{\prime}, K_{+1}{ }^{\prime}\right)$ of Enol I-acetylacetone.

Table S2: Measured Frequencies $v$ and deviations $\Delta v$ (observed-calculated) of $A_{1}$ state rotational transitions $J^{\prime}\left(K_{-1}{ }^{\prime}, K_{+1}{ }^{\prime}\right) \leftarrow J$ ” $\left(K_{-1}{ }^{\prime}, K_{+1}\right.$ ”) of the ${ }^{13} \mathrm{C}$ species of Enol I-acetylacetone.

Figures:

Figure S1: Keto I-acetylacetone

Figure S2: Keto II-acetylacetone 
Table S1: Measured Frequencies $v$ and deviations $\Delta v$ (observed-calculated) of $\mathrm{A}_{1}$ state rotational

transitions $J^{\prime}\left(K_{-1}{ }^{\prime}, K_{+1}{ }^{\prime}\right) \leftarrow J$ ” $\left(K_{-1}{ }^{\prime}, K_{+1}\right.$ ”) of Enol I-acetylacetone.

\begin{tabular}{|c|c|c|c|c|c|c|c|c|}
\hline \multicolumn{3}{|c|}{ MB-FTMW transitions (precision: 3 kHz) } & \multicolumn{6}{|c|}{ Millimeter-wave free jet transitions (precision: 100 kHz) } \\
\hline $\begin{array}{c}J^{\prime}\left(K_{-1}^{\prime}, K_{+1}^{\prime}\right) \leftarrow J ”\left(K_{-}\right. \\
\left.1^{\prime}, K_{+1} ”\right)\end{array}$ & v / MHz & $\Delta v / \mathrm{kHz}$ & $\begin{array}{c}J^{\prime}\left(K_{-1}{ }^{\prime}, K_{+1}{ }^{\prime}\right) \leftarrow J ”\left(K_{-}\right. \\
\left.1^{\prime}, K_{+1} ”\right)\end{array}$ & $v / \mathrm{MHz}$ & $\Delta v / \mathrm{kHz}$ & $J^{\prime}\left(K_{-1}^{\prime}\right) \leftarrow J^{\prime \prime}\left(K_{-1} "\right)^{\mathrm{a}}$ & $v / \mathrm{MHz}$ & $\Delta v / \mathrm{kHz}$ \\
\hline $1(1,1)-0(0,0)$ & 7382.9020 & 0.6 & $7(5,3)-6(4,2)$ & 61975.69 & 41 & $6(6)-5(5)$ & 67878.86 & -3 \\
\hline $2(1,2)-1(0,1)$ & 10084.2430 & -0.2 & $7(5,2)-6(4,3)$ & 61977.03 & 26 & $7(6)-6(5)$ & 70957.58 & 2 \\
\hline $2(2,1)-1(1,0)$ & 19447.0101 & 1.3 & $8(5,4)-7(4,3)$ & 65043.75 & -2 & $8(6)-7(5)$ & 74034.29 & 15 \\
\hline $2(2,0)-1(1,1)$ & 19842.7114 & 2.3 & $8(5,3)-7(4,4)$ & 65048.75 & 12 & $8(8)-8(7)$ & 67327.38 & 72 \\
\hline $3(1,3)-2(0,2)$ & 12608.3770 & 0.5 & $9(5,5)-8(4,4)$ & 68099.61 & -12 & $9(8)-9(7)$ & 67310.18 & -6 \\
\hline $3(2,2)-2(1,1)$ & 22148.1955 & 1.1 & $9(5,4)-8(4,5)$ & 68114.58 & -34 & $9(9)-9(8)$ & 76295.47 & 2 \\
\hline $3(2,2)-3(1,3)$ & 14617.0040 & 3.9 & $9(6,4)-8(5,3)$ & 77107.10 & -175 & $10(8)-10(7)$ & 67287.50 & -8 \\
\hline $3(2,1)-2(1,2)$ & 23380.9626 & -0.2 & $9(6,3)-8(5,4)$ & 77107.51 & -15 & $10(9)-10(8)$ & 76278.27 & -36 \\
\hline $3(2,1)-3(1,2)$ & 12497.5190 & -2.4 & $10(4,7)-9(3,6)$ & 61574.48 & 32 & 11(8)-11(7) & 67258.16 & 57 \\
\hline $3(3,1)-3(2,2)$ & 22488.9230 & 5.2 & $10(4,6)-9(3,7)$ & 62432.43 & 44 & 11(9)-11(8) & 76256.41 & 0 \\
\hline $3(3,0)-3(2,1)$ & 22374.7590 & -6.4 & $10(5,6)-9(4,5)$ & 71135.40 & -15 & $12(8)-12(7)$ & 67220.75 & 74 \\
\hline $4(1,4)-3(0,3)$ & 14985.3620 & -0.3 & $10(5,5)-9(4,6)$ & 71174.53 & 122 & 12(9)-12(8) & 76228.85 & 60 \\
\hline $4(2,3)-3(1,2)$ & 24659.9678 & 0.9 & $11(3,8)-10(2,9)$ & 61398.35 & -22 & $13(8)-13(7)$ & 67173.86 & 69 \\
\hline $4(2,3)-4(1,4)$ & 15387.5850 & 0.4 & $11(4,8)-10(3,7)$ & 64155.86 & 25 & $13(9)-13(8)$ & 76195.00 & 151 \\
\hline $4(3,2)-4(2,3)$ & 22550.1890 & 0.1 & $11(4,7)-10(3,8)$ & 65716.64 & 27 & $14(8)-14(7)$ & 67115.79 & -74 \\
\hline $4(3,1)-4(2,2)$ & 22215.3490 & -0.7 & $11(5,7)-10(4,6)$ & 74139.35 & 63 & $14(9)-14(8)$ & 76153.01 & -172 \\
\hline $5(1,5)-4(0,4)$ & 17261.7160 & -0.7 & $11(5,6)-10(4,7)$ & 74230.08 & 23 & $15(8)-15(7)$ & 67045.19 & 49 \\
\hline $5(1,4)-5(0,5)$ & 7893.3930 & -0.4 & $12(3,9)-11(2,10)$ & 66724.68 & -5 & & & \\
\hline $5(2,4)-5(1,5)$ & 16359.5440 & 1.9 & $12(4,9)-11(3,8)$ & 66501.36 & 27 & & & \\
\hline $5(2,3)-5(1,4)$ & 11574.7740 & 1.6 & $12(4,8)-11(3,9)$ & 69148.17 & 10 & & & \\
\hline $5(3,3)-5(2,4)$ & 22671.5180 & -2.5 & $12(5,8)-11(4,7)$ & 77093.74 & 75 & & & \\
\hline $5(3,2)-5(2,3)$ & 21917.7860 & 0.5 & $12(5,7)-11(4,8)$ & 77287.12 & 80 & & & \\
\hline $6(0,6)-5(1,5)$ & 15387.0824 & -0.9 & $13(3,10)-12(2,11)$ & 72638.69 & -34 & & & \\
\hline $6(1,6)-5(0,5)$ & 19494.3676 & 0.4 & $13(4,10)-12(3,9)$ & 68569.23 & 23 & & & \\
\hline $6(2,5)-6(1,6)$ & 17534.5050 & -1.4 & $13(4,9)-12(3,10)$ & 72793.15 & -17 & & & \\
\hline $6(2,4)-6(1,5)$ & 11289.4708 & -1.2 & $14(4,11)-13(3,10)$ & 70338.27 & -27 & & & \\
\hline $6(3,4)-6(2,5)$ & 22878.1470 & -2.0 & $14(4,10)-13(3,11)$ & 76731.35 & -5 & & & \\
\hline $6(3,3)-6(2,4)$ & 21448.2669 & -1.5 & $15(4,12)-14(3,11)$ & 71812.14 & 0 & & & \\
\hline $7(0,7)-6(1,6)$ & 18662.2057 & -0.1 & $16(4,13)-15(3,12)$ & 73016.43 & -23 & & & \\
\hline $7(1,7)-6(0,6)$ & 21739.3834 & 0.5 & $17(4,14)-16(3,13)$ & 73993.16 & -33 & & & \\
\hline
\end{tabular}

${ }^{\text {a }}$ Transitions doubly overlapped due to near prolate degeneracy of the involved levels. Only $K_{\mathrm{a}}$ is given. 
Table S2: Measured Frequencies $v$ and deviations $\Delta v$ (observed-calculated) of $\mathrm{A}_{1}$ state rotational transitions $J^{\prime}\left(K_{-1}{ }^{\prime}, K_{+1}{ }^{\prime}\right) \leftarrow J$ ” $\left(K_{-1}{ }^{\prime}, K_{+1}\right.$ ” $)$ of the ${ }^{13} \mathrm{C}$ species of Enol I-acetylacetone.

\begin{tabular}{|c|c|c|c|c|c|c|}
\hline \multirow{2}{*}{$\begin{array}{c}J^{\prime}\left(K_{-1}^{\prime}, K_{+1}{ }^{\prime}\right) \leftarrow J ”(K \\
\left.1^{\prime}, K_{+1} ”\right)\end{array}$} & \multicolumn{2}{|c|}{${ }^{13} \mathrm{C}_{\mathrm{CH} 3}$} & \multicolumn{2}{|c|}{${ }^{13} \mathrm{C}_{\mathrm{CO}}$} & \multicolumn{2}{|c|}{${ }^{13} \mathrm{C}_{\mathrm{CH}}$} \\
\hline & v/ MHz & $\Delta v / \mathrm{kHz}$ & v / MHz & $\Delta v / \mathrm{kHz}$ & v / MHz & $\Delta v / \mathrm{kHz}$ \\
\hline $1(1,1)-0(0,0)$ & 7311.6708 & 0.7 & 7375.6940 & 3.0 & 7334.3564 & -4.2 \\
\hline $2(1,2)-1(0,1)$ & 9963.1503 & 0.2 & 10066.4246 & 1.4 & 10031.1482 & -2.8 \\
\hline $3(1,3)-2(0,2)$ & 12442.7986 & -0.3 & 12581.4137 & -1.2 & 12549.7829 & -2.0 \\
\hline $3(2,1)-3(1,2)$ & 12479.0692 & 0.1 & 12520.0777 & -1.3 & 12358.0508 & 3.6 \\
\hline $4(0,4)-3(1,3)$ & 8343.3728 & 2.7 & 8526.7674 & 0.2 & 8627.1839 & -1.3 \\
\hline $4(1,4)-3(0,3)$ & 14778.8795 & -0.4 & 14950.1620 & -0.4 & 14921.0116 & -3.7 \\
\hline $5(0,5)-4(1,4)$ & 11705.1352 & 1.0 & 11938.1686 & -1.1 & 12046.2933 & 0.8 \\
\hline $5(1,5)-4(0,4)$ & 17015.2450 & 0.6 & 17218.3513 & 0.6 & 17192.3569 & 3.5 \\
\hline $6(0,6)-5(1,5)$ & 15018.8506 & -3.0 & 15297.5240 & 0.2 & 15408.5568 & 2.4 \\
\hline
\end{tabular}


Figure S1: Keto I-acetylacetone

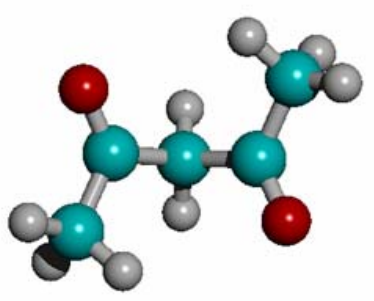

Figure S2: Keto II-acetylacetone

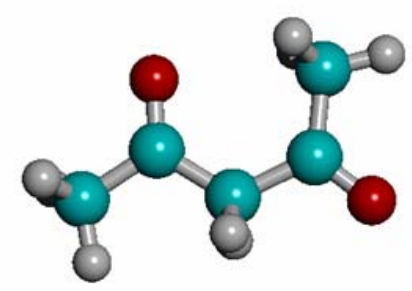

